\title{
Pico de torque e relação isquiotibiais/quadríceps de idosas praticantes de ginástica em dois grupos de convivência no contexto Passo Fundo - RS
}

\author{
Letícia Lopes", Ângelo Bonaldo Dalmut", Leonardo Siqueira de Azevedo"**, \\ Viviane Valério"***, Nilciane Coppatti"*** Cleiton Chiamonti Bona ${ }^{* * * *}$
}

\section{Resumo}

O principal objetivo desta pesquisa foi analisar os efeitos das aulas de ginástica sobre os músculos flexores e extensores da coxa de 68 idosas, com idade superior a sessenta anos, pertencentes aos grupos do Creati e do Dati, dos bairros São José, Santo Antônio e Jaboticabal do município de Passo Fundo. Para tanto, observou-se o equilíbrio muscular, visando à prevenção contra possíveis quedas e lesões. Com o uso do dinamômetro isocinético da marca Biodex ${ }^{\mathrm{TM}}$, modelo Multi Joint System 3 Pro computadorizado, disponibilizado no Laboratório de Biomecânica da Universidade de Passo Fundo, efetivou-se a medida de força máxima dos músculos da coxa em movimento de flexão e extensão, nas velocidades angulares de $60 \%$, $180 \%$ s e $240 \%$, através do pico de torque $\left(\mathrm{P}_{\mathrm{T}}\right)$. Foram demonstrados desequilíbrios musculares de força, considerando a rela- ção isquiotibiais/quadríceps $\left(R_{\mathrm{v} / \mathrm{Q}}\right)$, e analisados os resultados com a aplicação do teste " $\mathrm{t}$ " de Student, utilizando-se $\mathrm{p}<0,05$. Constatou-se que, nos resultados de $\mathrm{P}_{\mathrm{T}} \mathrm{O}$ Dati obteve média estatisticamente maior em comparação ao Creati na extensão do joelho direito e esquerdo em $180^{\circ}$ s e na extensão do joelho esquerdo na velocidade de $120 \%$ s. Ambos, na $\mathrm{R}_{\mathrm{I} / \mathrm{Q}^{\prime}}$ obtiveram resultados inferiores a $60 \%$ em menores velocidades e, em velocidade de $240 \%$ s, alcançaram o valor citado, o que evidencia o desequilíbrio muscular. Percebeu-se a necessidade de pesquisas nesta área para melhor objetivar as aulas para idosos, pois a infraestrutura do local das atividades não constitui fator decisivo para o desenvolvimento do equilíbrio muscular.

Palavras-chave: Idosos. Dinamômetro de força muscular. Torque.

* Graduada em Educação Física pela Faculdade de Educação Física e Fisioterapia da Universidade de Passo Fundo. Pós-graduanda em Treinamento Esportivo na Universidade de Passo Fundo. Endereço para correspondência: Rua Silva Jardim, 277, centro, CEP: 99010-130, Passo Fundo - RS. E-mail: leticialopes@yahoo. com.br.

* Graduado em Educação Física pela Faculdade de Educação Física e Fisioterapia da Universidade de Passo Fundo. Pós-graduando em Treinamento Esportivo na Universidade de Passo Fundo.

**** Graduados em Educação Física pela Faculdade de Educação Física e Fisioterapia, Universidade de Passo Fundo.

**** Mestre em Ciências do Movimento Humano pela Universidade do Estado de Santa Catarina. Professor da Faculdade de Educação Física e Fisioterapia da Universidade de Passo Fundo.

$\rightarrow$ Recebido em maio de 2009 - Avaliado em março de 2010.

$\rightarrow$ doi:10.5335/rbceh.2010.005 


\section{Introdução}

O envelhecimento humano, dentro de condições adequadas de sobrevivência, é um processo condicional a todos. (ALVES et al., 2004). Em tempos atuais, avalia-se o aumento da população de idosos, havendo, por parte de pesquisadores, maior preocupação e interesse no que se refere a debilidades existentes nessa faixa etária. (MAZO et al., 2007).

Constata-se que, em razão de alterações biológicas, psicológicas e sociais (BENEDETTI; BENEDETTI, 1996), os idosos acabam passando por incapacidades funcionais que podem dificultar as tarefas do cotidiano (MAZO et al., 2007) e influenciar no relacionamento do idoso consigo mesmo e com os demais sujeitos do seu meio social. (BENEDETTI; BENEDETTI,1996).

Com as limitações, ocorre o risco de possíveis doenças crônico-degenerativas ligadas ao sedentarismo, que, no caso de uma doença cardiovascular, podem levar à morte. (KALACHE et al. apud ALVES et al., 2004).

Considerando o processo de envelhecimento e a existência de alterações, em especial das fisiológicas, no sistema neuromuscular ocorrem a diminuição da força muscular, o aumento da perda da densidade óssea mineral e, por fim, a instabilidade postural, que leva muitas vezes a quedas e fraturas. (DAVINI; NUNES, 2003; MAZO et al., 2007).

Segundo Nadeau e Peronnet (1985, apud BENEDETTI, 1996), a prática de exercícios traz vários benefícios aos idosos, como o aumento da massa muscular e da força, a diminuição do percentual de gordura corporal, o nivelamento da pressão sanguínea e da frequência cardíaca conforme a idade, dificultando o acúmulo de colesterol sanguíneo e facilitando a locomoção. No entanto, mesmo com tantos benefícios à terceira idade, o motivo pela busca por exercícios ou atividades físicas em uma idade mais avançada está ligado à necessidade de controlar o peso, evitar o risco de doenças, diminuir o estresse e a depressão, melhorar a autoestima e proporcionar a socialização. (WEINBERG; GOULD, 2001; CAROMANO; IDE; KERBAUY, 2006).

Além disso, por meio de pesquisas, Lampinem et al. (apud MORAES et al., 2007) informam que, após a diminuição de atividades físicas por um período de oito anos, idosos apresentaram aumento dos sintomas de depressão, o que não ocorreu com idosos que mantiveram suas práticas.

Vários estudos fundamentam a necessidade do exercício físico regular para o idoso, pois se sabe que essa prática pode melhorar a força muscular, a agilidade (reação), o equilíbrio, a velocidade de marcha e, até mesmo, o desenvolvimento cognitivo (GUIMARÃES; CALDAS, 2006), da mesma forma que promove a minorização do declínio da aptidão cardiorrespiratória, contribuindo para uma vida mais independente. (KRAUSE et al., 2007).

Seguindo-se esse pensamento, no município de Passo Fundo - RS existem vários grupos de idosos, dos quais serão destacados o Centro Regional de Estudo e Atividades para Terceira Idade (Creati) e o Departamento de Atendimento à Terceira Idade (Dati), que oferecem diversas 
oficinas, incluindo as de ginástica. Após a exposição do panorama de atendimento fornecido a esses grupos, existe a necessidade de tratar sobre o desenvolvimento de força muscular, pois essa capacidade é determinante no auxílio de diversas práticas. (CARVALHO et al., 2004).

Sobre o tema, afirmam Brill et al. (apud CARVALHO et al., 2004): "A perda da força e da massa muscular predispõe os idosos a uma limitação funcional, sendo este um fator predisponente para muitos dos processos patológicos associados ao aumento da morbilidade e mortalidade".

A força muscular é uma capacidade motora muito reduzida no idoso, pois, com o passar dos anos, há uma diminuição de área da secção transversa (AST) e, consequentemente, a atrofia muscular, incluindo o recrutamento e o disparo de unidades motoras. No entanto, sabe-se que, adotando-se uma atividade que vise ao ganho de força muscular em idosos, existe a evidência de adaptações neuromusculares desses indivíduos, pela verificação do aumento da AST após o período de treinamento. (DAVINI; NUNES, 2003).

No que se refere às atividades generalizadas de ginástica para idosos, apesar da sua grande oferta, não existem muitas pesquisas quanto a seus resultados. (LORD et al. apud CARVALHO et al., 2004). Desse modo, a fim de contribuir para a diminuição dessa lacuna, por meio de uma avaliação isocinética de membros inferiores, foram verificados o equilíbrio muscular e o padrão funcional de força. (ASSIS; GOMES; CARVALHO, 2005). Esse método, em razão de sua na- tureza reprodutível e objetiva, é utilizado em estudos de desempenho muscular com idosos, prevenindo possíveis quedas ou lesões, bem como indicando diferentes dados normativos para diferentes condicionamentos físicos e faixas etárias. (NEDER et al., 1999; CARVALHO et al., 2003).

Nas avaliações no dinamômetro isocinético, a verificação de equilíbrio e padrão funcional de força muscular dos membros inferiores foi realizada pela medição do peak torque $\left(\mathrm{P}_{\mathrm{T}}\right)$, ou seja, do ponto de maior torque (momento de força) aplicado durante a amplitude do movimento, realizando a relação entre isquiotibiais e quadríceps $\left(\mathrm{R}_{\mathrm{I} / \mathrm{Q}}\right)$ dos membros dominantes e não dominantes. Nessa relação entre os músculos da coxa - $R_{\mathrm{I} / \mathrm{Q}}$, também entendida em algumas pesquisas como agonistas e antagonistas, flexores e extensores, analisa-se, de acordo com os parâmetros, a harmonia de tais músculos, quantificando valores absolutos do torque, do trabalho e da potência de grupos musculares expostos em porcentagens.

É importante salientar que normalmente se coloca o valor de $60 \%$ para o $\mathrm{P}_{\mathrm{T}}$ entre os flexores/extensores, resultando na diferença de $40 \%$ entre os extensores (mais fortes) e os flexores (mais fracos). (TERRERI; GREVE; AMATUZZI, 2001; BITTENCOURT et al., 2005). Frequentemente, assimetrias ou défices na produção de torque máximo entre membros acima de $10 \%$ têm sido associados a lesões musculares. (PERRIN; ROBERTSON, RAY apud FONSECA et al., 2007; TERRERI; GREVE; AMATUZZI, 2001). 
Nesta pesquisa procurou-se avaliar se as idosas que recebem atendimento no seu próprio bairro, normalmente com infraestrutura inferior à oferecida pela universidade, e que realizam atividades que se equivalem às dessa instituição em relação à força de membros inferiores. No estudo analisou-se a diferença do $\mathrm{P}_{T}$ na $R_{\mathrm{I} / \mathrm{Q}}$ (relação isquiotibiais e quadríceps), verificando o equilíbrio muscular.

\section{Material e métodos}

A presente pesquisa avaliou o $\mathrm{P}_{\mathrm{T}}$ na $R_{\mathrm{I} / \mathrm{Q}}$ de idosas participantes de grupos de terceira idade do município de Passo Fundo vinculados ao Creati e ao Dati. O Creati desenvolve suas atividades no prédio da Faculdade de Educação Física e Fisioterapia, em local e com materiais apropriados. O Dati, por sua vez, está vinculado à Secretaria Municipal de Cidadania e Assistência Social, da Prefeitura Municipal de Passo Fundo, oferecendo atendimento nos bairros da cidade, em salões comunitários ou ginásios escolares (conforme a comunidade dispõe), sendo os materiais obtidos pela participação dos alunos inseridos nos grupos.

Nesta pesquisa, foram avaliadas as idosas participantes da Dati que moram nos bairros São José, Santo Antônio e Jaboticabal. As aulas de ginástica ocorrem em ambos os grupos duas vezes por semana, com duração, em média, de 1 hora/aula. As atividades são diversificadas, havendo recreações, danças, alongamentos, exercícios de reforço muscular (sem e com peso) e, até mesmo, momentos de conversação. Para a pesquisa, as interessadas em participar receberam um termo de consentimento livre e esclarecido sobre a avaliação a ser realizada. O estudo está de acordo com as diretrizes da resolução 196/1996 do Conselho Nacional da Saúde, tendo obtido aprovação pelo Comitê de Ética em Pesquisa da Universidade de Passo Fundo sob registro $n^{\circ} 127 / 2008$.

Participaram do estudo 68 idosas com idade igual ou superior a sessenta anos, conforme o próprio regulamento desses grupos de atendimento a idosos estabelece como faixa etária. Como critérios de exclusão da amostra foram definidos os sujeitos que apresentaram grandes instabilidades articulares ou cardíacas que pudessem ser agravadas durante os testes, bem como as idosas que não concordaram em assinar o termo de consentimento livre e esclarecido, ou as que em algum momento desistiram de participar da pesquisa.

A avaliação para a coleta de dados ocorreu no Laboratório de Biomecânica da Universidade de Passo Fundo, junto à Faculdade de Educação Física e Fisioterapia, estando cada idosa presente no dia e horário previamente marcados. Optou-se por manter sempre o mesmo avaliador para os testes isocinéticos, com vistas a garantir a padronização e o controle. A avaliação isocinética do $\mathrm{P}_{\mathrm{T}}$ foi realizada com o auxílio de dinamômetro da marca Biodex ${ }^{\mathrm{TM}}$ modelo Multi Joint System 3 Pro computadorizado.

Inicialmente, as idosas foram apresentadas aos materiais que utilizariam para, em seguida, realizarem um alongamento passivo de membros inferiores e, posteriormente, um aquecimento 
em bicicleta ergométrica Movement Technology ${ }^{\circledR}$ BM 2700, sem carga, durante cinco minutos.

Na medição do movimento de extensão e flexão do joelho, as participantes sentaram-se na cadeira do equipamento, apoiando as costas no encosto a $85^{\circ}$, para ser ajustado até que a fossa poplítea estivesse apoiada na parte anterior do assento. Estabilizou-se o tronco com cintos de segurança colocados em volta da cintura, dois cruzando as espinhas ilíacas anterossuperiores, fixando a coxa com uma cinta de velcro que passava acima da articulação do joelho e $2 \mathrm{~cm}$ acima do maléolo medial, para estabilizar a perna. O eixo de rotação do dinamômetro foi alinhado com o eixo da articulação do joelho, conforme descrito no manual de aplicações e operações Biodex ${ }^{\mathrm{TM}}$ Multi Joint System 3 Pro.

Para melhor adaptação com o dinamômetro isocinético, as participantes efetuaram três movimentos livres antes de iniciar a mensuração, que seguiu o protocolo de avaliação composto por três velocidades angulares: $120 \%$ s, $180 \% / \mathrm{s} \mathrm{e}$ $240 \%$ s. Cada idosa executou cinco movimentos completos em cada uma das velocidades, partindo da posição de flexão máxima. Após cada uma das séries era permitido um intervalo de $30 \mathrm{~s}$. Os procedimentos foram aplicados, primeiramente, em um dos membros inferiores e, em seguida, no outro.
Para a análise da $R_{\mathrm{I} / \mathrm{Q}}$ foi ocupado o $\mathrm{P}_{\mathrm{T}}$ dos músculos flexores do joelho (isquiotibiais) pelo $\mathrm{P}_{\mathrm{T}}$ dos músculos extensores do joelho (quadríceps), multiplicados por 100 (BITTENCOURT et al.,2005), tomando-se como referência de porcentagem o valor de $60 \%$ para o $\mathrm{P}_{\mathrm{T}}$ entre os flexores/extensores (PERRIN; ROBERTSON; RAY apud FONSECA et al., 2007; TERRERI; GREVE; AMATUZZI, 2001). Os dados do $\mathrm{P}_{\mathrm{T}}$ foram verificados por meio dos recursos da análise descritiva e, para a comparação entre os grupos, procedeu-se ao teste $t$ de Student, utilizando-se $\mathrm{p}<0,05$ para todos os testes realizados.

Ao final dos testes, foram realizados alongamentos dos grupos musculares envolvidos, orientados pelos avaliadores. Posteriormente, a avaliação impressa foi entregue.

\section{Resultados}

$\mathrm{Na}$ comparação entre joelho direito e esquerdo no $\mathrm{P}_{\mathrm{T}}$ do grupo de idosas do Dati, conforme a Tabela 1 , não foram apresentados valores inferiores ao $p$ menor que 0,05 , demonstrando que, para esses sujeitos da pesquisa, não houve diferença significativa entre as forças do joelho direito em relação ao esquerdo. 
Tabela 1 - Média do $\mathrm{P}_{\mathrm{T}}$ do Dati e Creati do joelho direito e esquerdo.

\begin{tabular}{|c|c|c|c|c|c|}
\hline Grupo & Velocidade & Movimento & $\begin{array}{l}\text { Joelho direito } \\
\qquad(\mathrm{Nm})\end{array}$ & $\begin{array}{l}\text { Joelho esquerdo } \\
\qquad(\mathrm{Nm})\end{array}$ & $\mathrm{p}$ \\
\hline \multirow{6}{*}{ Dati } & \multirow{2}{*}{$120 \% \mathrm{~s}$} & Extensão & $67,14 \pm 18,91$ & $65,06 \pm 21,38$ & 0,194 \\
\hline & & Flexão & $31,54 \pm 9,49$ & $31,78 \pm 9,69$ & 0,423 \\
\hline & \multirow{2}{*}{$180^{\circ} / \mathrm{s}$} & Extensão & $56,81 \pm 16,03$ & $56,42 \pm 16,23$ & 0,4203 \\
\hline & & Flexão & $30,85 \pm 8,81$ & $31,04 \pm 8,76$ & 0,4364 \\
\hline & \multirow{2}{*}{$240 \% \mathrm{~s}$} & Extensão & $49,61 \pm 13,48$ & $48,87 \pm 12,71$ & 0,2661 \\
\hline & & Flexão & $30,52 \pm 8,77$ & $29,44 \pm 8,44$ & 0,1530 \\
\hline \multirow{6}{*}{ Creati } & \multirow{2}{*}{$120 \% \mathrm{~s}$} & Extensão & $59,15 \pm 18,32$ & $56,48 \pm 16,50$ & 0,0586 \\
\hline & & Flexão & $29,32 \pm 9,37$ & $28,19 \pm 9,91$ & 0,2043 \\
\hline & \multirow{2}{*}{$180^{\circ} / \mathrm{s}$} & Extensão & $49,68 \pm 13,82$ & $49,72 \pm 13,37$ & 0,4927 \\
\hline & & Flexão & $29,08 \pm 8,47$ & $28,13 \pm 9,54$ & 0,2778 \\
\hline & \multirow{2}{*}{$240 \% / \mathrm{s}$} & Extensão & $46,74 \pm 12,90$ & $44,37 \pm 11,00$ & 0,0803 \\
\hline & & Flexão & $32,19 \pm 10,44$ & $29,29 \pm 11,29$ & 0,0433 \\
\hline
\end{tabular}

Na comparação entre joelho direito demonstra que no lado direito os sujeie esquerdo no $\mathrm{P}_{\mathrm{T}}$ do grupo do Creati, a tos do Creati apresentam maior força maioria dos movimentos não apresentou no joelho $\left(\mathrm{P}_{\mathrm{T}}\right)$ em alta velocidade, pois, diferença estatística, somente na flexão quanto maior é a velocidade, menor é a do joelho na velocidade de $240 \%$ s, que resistência que o aparelho oferece, faciresultou num valor de $\mathrm{p}=0,043$. Isso litando o movimento realizado.

Tabela 2 - Média do $\mathrm{P}_{\mathrm{T}}$ dos joelhos comparando Dati e Creati.

\begin{tabular}{|c|c|c|c|c|c|}
\hline Joelho & Velocidade & Movimento & $\begin{array}{c}\text { Dati } \\
\text { Pico de torque } \\
(\mathrm{Nm})\end{array}$ & $\begin{array}{c}\text { Creati } \\
\text { Pico de torque } \\
(\mathrm{Nm})\end{array}$ & $P$ \\
\hline \multirow{6}{*}{ Direito } & \multirow{2}{*}{$120 \% \mathrm{~s}$} & Extensão & $67,14 \pm_{18,91}$ & $59,15 \pm_{18,32}$ & \multirow{2}{*}{$\begin{array}{l}0,048 \\
0,178\end{array}$} \\
\hline & & Flexão & $31,54 \pm 9,49$ & $29,32 \pm 9,37$ & \\
\hline & \multirow{2}{*}{$180^{\circ} / \mathrm{s}$} & Extensão & $56,81 \pm_{16,03}$ & $49,68 \pm_{13,82}$ & \multirow{2}{*}{$\begin{array}{l}0,035 \\
0,212\end{array}$} \\
\hline & & Flexão & $30,85 \pm 8,81$ & $29,08 \pm 8,47$ & \\
\hline & \multirow{2}{*}{$240^{\circ} / \mathrm{s}$} & Extensão & $49,61 \pm_{13,48}$ & $46,74 \pm_{12,90}$ & \multirow{2}{*}{$\begin{array}{l}0,198 \\
0,243\end{array}$} \\
\hline & & Flexão & $30,52 \pm 8,77$ & $32,19 \pm 10,44$ & \\
\hline \multirow{6}{*}{ Esquerdo } & \multirow{2}{*}{$120 \% \mathrm{~s}$} & Extensão & $65,06 \pm 21,38$ & $56,48 \pm 16,50$ & \multirow{2}{*}{$\begin{array}{l}0,046 \\
0,075\end{array}$} \\
\hline & & Flexão & $31,78 \pm 9,69$ & $28,19 \pm 9,91$ & \\
\hline & \multirow{2}{*}{$180^{\circ} / \mathrm{s}$} & Extensão & $56,42 \pm_{16,23}$ & $49,72 \pm 13,37$ & \multirow{2}{*}{0,104} \\
\hline & & Flexão & $31,04 \pm 8,76$ & $28,13 \pm 9,54$ & \\
\hline & \multirow{2}{*}{$240 \% / s$} & Extensão & $48,87 \pm_{12,71}$ & $44,37 \pm 11,00$ & \multirow{2}{*}{$\begin{array}{l}0,074 \\
0,476\end{array}$} \\
\hline & & Flexão & $29,44 \pm 8,44$ & $29,29 \pm_{11,29}$ & \\
\hline
\end{tabular}


Analisando a Tabela 2, na qual é comparado o joelho direito dos idosos do Dati com o joelho direito dos idosos do Creati, percebe-se maior média nos movimentos e velocidades executadas pelo Dati. Todavia, a única diferença estatística obtida ocorreu na extensão em $180 \%$ s no joelho direito, que resultou um $\mathrm{p}=0,035$.

Observando-se essa mesma tabela, porém no lado esquerdo, os sujeitos do Dati também obtiveram média maior em relação aos do Creati, mas somente nas velocidades de $120 \%$ s e $180 \%$ s, nos movimentos de extensão do joelho esquerdo, que obteve um valor de $\mathrm{p}$ significativo, respectivamente, 0,046 e 0,044 .

Portanto, na maioria dos movimentos e velocidades executados, comparando grupos de terceira idade, o Dati obteve média levemente maior que a do Creati. No entanto, estatisticamente, somente na extensão em $180^{\circ}$ /s no joelho direito e esquerdo o Dati foi superior e, também no joelho esquerdo, na velocidade de $120 \%$ s.

Tabela 3 - Relação I/Q do Dati e Creati do joelho direito e esquerdo.

\begin{tabular}{llcccc}
\hline Joelho & Velocidade & $\begin{array}{c}\text { Dati } \\
\text { Ísquio/Quadríceps }\end{array}$ & $\begin{array}{c}\mathrm{R}=\mathrm{I} / \mathrm{Q} \\
\%\end{array}$ & $\begin{array}{c}\text { Creati } \\
\text { Ísquio/Quadríceps }\end{array}$ & $\begin{array}{c}\mathrm{R}=\mathrm{I} / \mathrm{Q} \\
\%\end{array}$ \\
\hline \multirow{3}{*}{ Direito } & $120 \% \mathrm{~s}$ & $31,54 / 67,14$ & 46,97 & $29,32 / 59,15$ & 49,56 \\
& $180 \% \mathrm{~s}$ & $30,85 / 56,81$ & 54,30 & $29,08 / 49,68$ & 58,53 \\
& $240 \% \mathrm{~s}$ & $30,52 / 49,61$ & 61,51 & $32,19 / 46,74$ & 68,87 \\
\multirow{3}{*}{ Esquerdo } & $120 \% \mathrm{~s}$ & $31,78 / 65,06$ & 48,84 & $28,19 / 56,48$ & 49,91 \\
& $180 \% \mathrm{~s}$ & $31,04 / 56,42$ & 55,01 & $28,13 / 49,72$ & 56,57 \\
& $240 \%$ & $29,44 / 48,87$ & 60,24 & $29,29 / 44,37$ & 66,01 \\
\hline
\end{tabular}

$\mathrm{AR}_{\mathrm{I} / \mathrm{Q}}$ dentro dos $60 \%$ equivale a um bom equilíbrio muscular entre flexores e extensores. Verifica-se na Tabela 3, a $R_{\mathrm{I} / \mathrm{Q}}$, que nos joelhos direito e esquerdo dos dois grupos, nas velocidades de $120 \%$ s e $180 \%$ s, nem o Dati nem o Creati alcançaram a referida porcentagem; somente na maior velocidade, ou seja, de $240 \%$ s, foi possível alcançá-la, o que demonstra o desequilíbrio muscular em velocidades nas quais o aparelho oferece maior resistência ao movimento.

\section{Discussão}

Apesar de, no princípio da pesquisa, ter havido o indicativo de que as idosas participantes do grupo do Creati alcançariam resultados, na sua maioria, superiores, em virtude da melhor infraestrutura e da qualidade dos materiais que têm à sua disposição, com a realização deste estudo pôde-se verificar o contrário, pois foi o Dati que, em alguns dados, obteve resultados maiores e também estatisticamente comprovados para o $\mathrm{P}_{\mathrm{T}}$.

Sugere-se que tais resultados se devam ao estilo de vida dos sujeitos pesquisados, pois, ao longo da sua existência, os indivíduos integrantes do Dati realizaram atividades diárias que envolvem maiores gastos energéticos. Sendo, em grande parte, integrantes de uma classe social menos favorecida, executaram, ou 
ainda executam, trabalhos que requerem esforço físico, o que favorece o desenvolvimento de maior resistência muscular. (CARVALHO et al., 2004).

Em contrapartida, os integrantes do Creati relataram ter exercido atividades relacionadas ao ensino, como é o caso de professoras, ou somente relativas à organização de suas residências, as quais exigem menor resistência de força. $\mathrm{Da}$ mesma maneira, geralmente as participantes do Dati realizam o deslocamento para exercer suas atividades caminhando, ao passo que algumas participantes do Creati dispõem de outros meios de locomoção.

Analisando a questão da faixa etária, o Dati possui média de $66,20 \pm 5,42$ e o Creati, $69,68 \pm 5,40$, o que pode indicar a razão dos resultados de torque, com diferença favorável ao Dati.

Na obtenção de respostas mais específicas para tais resultados, por meio do diálogo com os professores responsáveis pelos grupos, ficou esclarecido que as alunas do Creati não costumam realizar movimentos de flexão e extensão em velocidades maiores em seus exercícios, permanecendo, normalmente, com movimentos lentos.

Com base nos resultados do $\mathrm{P}_{\mathrm{T}}$ na realização da $R_{\mathrm{I} / \mathrm{Q}}$ pode-se destacar o desequilíbrio muscular encontrado nos grupos do Dati e do Creati nas velocidades menores $(120 \%$ s e $180 \%$ s), as quais oferecem maior resistência ao movimento, exigindo maior força. (TERRERI; GREVE; AMATUZZI, 2001). Desse modo, verificou-se um resultado alarmante e, ao mesmo tempo, instigante, pois tal constatação sugere que ambos os grupos apresentam deficiências musculares, ou, por outro lado, talvez o parâmetro citado pela literatura não seja ideal para idosos, havendo a necessidade de um maior número de pesquisas na área para que seja encontrado um indicador adequado. (BITTENCOURT et al. 2005; CARVALHO et al., 2003).

Neste estudo, o resultado apresentado demonstra, dentro dos parâmetros indicados, a existência de desequilíbrio muscular, avaliando-se a necessidade de exercícios que ofereçam força aos músculos quadríceps e ísquios-tibiais, melhorando a eficiência dos membros inferiores, a fim de reduzir o número de quedas ou lesões entre idosos. (DAVINI; NUNES, 2003).

Evidenciando os valores na velocidade de $240 \%$, que oferece menor resistência em sua realização, os dois grupos de idosas, ao contrário dos resultados obtidos anteriormente, alcançaram o parâmetro referido pela literatura. Esse fato sugere que movimentos que exigem menos esforço favorecem o alcance dos índices citados. (TERRERI; GREVE; AMATUZZI, 2001).

\section{Conclusão}

Com os resultados desta pesquisa ficou evidenciada a importância de sua execução para os profissionais da área de saúde, em especial para os professores de educação física, que poderão objetivar suas práticas com os idosos visando à melhora da harmonia muscular. Do mesmo modo, confirmou-se a necessidade de maiores investigações e pesquisas sobre o exercício físico e o idoso. 
Desse modo, será esclarecida aos acadêmicos de educação física e aos próprios idosos a importância de exercícios envolvendo essa faixa etária, a qual requer aulas que proporcionem, de maneira estruturada, a melhora da qualidade e a perspectiva de vida saudável.

Nesse sentido, foi observado que o local em que ocorrem as aulas e o material disponível, no caso desta pesquisa, não influenciaram de maneira decisiva na melhora da performance muscular das idosas, mas, sim, a maneira como realizam seus exercícios e suas tarefas do cotidiano. Por fim, confirma-se a necessidade de busca por mais conhecimentos que envolvam a faixa etária estudada. colleges. At Biomechanics Laboratory, it was carried out the measure of maximum strength on ischium-tibial muscles and quadriceps in flexion and stretching movements, at angular velocities of $60 \% \mathrm{~s}, 180 \% \mathrm{~s}$ and $300 \%$, through Torque Peak(Pt), showing muscular instabilities of strength by using deficits shown by ischium-tibials/ quadriceps (RI/q). However, Dati got an average slightly bigger than Creati, nevertheless, statically, only on right and left knee extension at $180 \%$ s and in extension in velocity of $120^{\circ} / \mathrm{s}$ Dati was superior. It proves that independent of infra-structure that these groups have, it's possible that the subjects own balanced strength on inferior limbs.

Key words: Aged. Muscle strength dynamometer. Torque.

\section{Torque peak and i/q relationship of elderly students doing physical exercises at Creati and Dati}

\section{Abstract}

Several research and studies about regular physical exercise are beneficial to physical skill, but a few ones are aimed at The Elderly, that's why this project has as main objective the analysis of effects of work out classes generalized over flexor and extensor muscles of the thigh of 68 elderly women being senior citizen, Creati and Dati, from São José, Santo Antonio and Jaboticaball neighborhoods, over 60 years old, in Passo Fundo city, comparing the muscular stability between inferior limbs, because when they are debilitated, they may bring about collapses, injuries and even prevent the elderly from doing their activities. By using Biodex isokinetic dynamometer, Multi Joint System 3 Pro computerized model, provided by Passo Fundo University, with Physical Education and Physiotherapy

\section{Agradecimentos}

À Universidade de Passo Fundo, por meio da Faculdade de Educação Física e Fisioterapia, a qual me disponibilizou não só o seu curso de pós-graduação em Treinamento Esportivo, mas também toda sua infraestrutura, professores, alunos e funcionários. Agradeço também ao grupo de graduandos em Educação Física da UPF que auxiliaram nas avaliações das idosas.

\section{Referências}

ALVES, R. V. et al. Aptidão física relacionada à saúde de idosos: influência da hidroginástica. Revista Brasileira de Medicina do Esporte, Niterói, v. 10, n. 1, p. 1-9, jan./fev. 2004.

ASSIS, M. M. V.; GOMES, M. I.; CARVALHO, E. M. S. Avaliação isocinética de quadríceps e ísquios-tibiais nos atletas de jiu-jitsu. $R e$ vista Brasileira em Promoção da Saúde, São Paulo, v. 18, n. 2, p. 85-89, mar. 2005. 
BENEDETTI, T. R. B.; BENEDETTI, A. L. Musculação na terceira idade. Revista de Educação Física / UEM, Rio de Janeiro, v. 7, n. 1, p. 35-40, 1996.

BITTENCOURT, N. F. N. et al. Avaliação muscular isocinética da articulação do joelho em atletas das seleções brasileiras infanto e juvenil de voleibol masculino. Revista Brasileira de Medicina do Esporte, Niterói, v. 11, n. 6, p. 331-336, nov./dez. 2005.

CAROMANO, F. A.; IDE, M. R.; KERBAUY, R. R. Manutenção na prática de exercícios por idosos. Revista do Departamento de Psicologia/UFF, Niterói, v. 18, n. 2, p. 1-9, jul./dez. 2006.

CARVALHO, J. et al. Efeito de um programa de treino em idosos: comparação da avaliação isocinética e isotônica. Revista Paulista de Educação Física, São Paulo, v. 17, n. 1, p. 74-84, jan./jun. 2003.

CARVALHO, J. et al. Força muscular em idosos 1: Será o treino generalizado suficientemente intenso para promover o aumento da força muscular em idosos de ambos os sexos?. Revista Portuguesa de Ciências do Desporto, v. 4, n. 1, p. 51-57, jun. 2004.

CARVALHO, P.; CABRI, J. Avaliação isocinética da força dos músculos da coxa em futebolistas. Revista Portuguesa de Fisioterapia no Desporto, v. 1, n. 21, p. 4-13, jul. 2007.

DAVINI, R.; NUNES, C. V. Alterações no sistema neuromuscular decorrentes do envelhecimento e o papel do exercício físico na manutenção da força muscular em indivíduos idosos. Revista Brasileira de Fisioterapia, São Carlos, v. 7, n. 3, p. 201-207, set./dez. 2003.

FONSECA, S. T. da et al. Caracterização da performance muscular em atletas profissionais de futebol. Revista Brasileira de Medicina do Esporte, Niterói, v. 13, n. 3, mai./jun. 2007.

GOULART, L. F.; DIAS, R. M. R.; ALTIMARI, L. R. Variação do equilíbrio muscular durante uma temporada em jogadores de futebol categoria sub-20. Revista Brasileira de Medicina do Esporte, Niterói, v. 14, n. 1, p. 17-21, jan./fev. 2008.

GUIMARÃES, J. M. N.; CALDAS, C. P. A influência da atividade física nos quadros depressivos de pessoas idosas: uma revisão sistemática. Revista Brasileira de Epidemiologia, São Paulo, v. 9, n. 4, p. 1-9, dez. 2006.

KRAUSE, M. P. et al. Influência do nível de atividade física sobre a aptidão cardiorrespiratória em mulheres idosas. Revista Brasileira de Medicina do Esporte, Niterói, v. 13, n. 2, p. 1-10, mar./abr. 2007.

MAZO, G. Z. et al. Condições de saúde, incidência de quedas e nível de atividade física dos idosos. Revista Brasileira de Fisioterapia, São Carlos, v. 11, n. 6, p. 1-7, nov./dez. 2007.

MORAES, H. et al. O exercício físico no tratamento da depressão em idosos: revisão sistemática. Revista de Psiquiatria do Rio Grande do Sul, Porto Alegre, v. 29, n. 1, p. 1-8, jan./abr. 2007.

NEDER, J. A. et al. Reference values for concentric knee isokinetic strength and power in nonathletic men and women from 20 to 80 years old. Journal of Orthopedic \& Sports Physical Therapy, v. 29, p. 116-26, 1999.

TERRERI, A. S. A. P.; GREVE, J. M. D.; AMATUZZI, M. M. Avaliação isocinética no joelho do atleta. Revista Brasileira de Medicina do Esporte, Niterói, v. 7, n. 5, p. 170-174, set./out. 2001.

WEINBERG, R. S.; GOULD, D. Fundamentos da psicologia do esporte e do exercício. 2. ed. Porto Alegre: Artmed, 2001. 